\title{
Safety Zones and Standards in Magnetic Resonance Imaging
}

\author{
Vitor Silva ${ }^{1 *}$, Isabel Ramos², J Agostinho Moreira ${ }^{3}$ and Manuel B Marques ${ }^{4}$ \\ ${ }^{1}$ Magnetic Resonance Imaging, Department Centro Hospitalar e Universitario Sao Joao, EPE, Oporto, Portugal. \\ ${ }^{2}$ Faculty of Medicine, University of Porto, Portugal
}

${ }^{3}$ Department of Physics and Astronomy, IFIMUP and IN-Institute of Nanoscience and Nanotechnology, Faculty of Sciences, University of Porto.

${ }^{4}$ Department of Physics and Astronomy, Faculty of Sciences, University of Porto; INESC-TEC, Institute of Computer Systems Engineering - Technology and Science

*Corresponding author: Vitor Silva, Magnetic Resonance Imaging, Department Centro Hospitalar e Universitario Sao Joao, EPE, Oporto, Portugal.
Received Date: January 04, 2021

Published Date: January 21, 2021

\begin{abstract}
Magnetic resonance imaging is a medical technique widely accepted in Medicine. It raises new questions and paradigms to safety standards. Magnetic resonance imaging uses three types of electromagnetic fields for its operation: a static magnetic fields, time-varying gradient fields and radiofrequencies fields. With new technology, static magnetic fields used in magnetic resonance will increase its field strength. So, the introduction of safety zones and standards in magnetic resonance is vital for better practices. American College of Radiology introduced four conceptual safety zones with personnel entrance restrictions Zone 1 includes areas freely accessed. Zone 2 is where patients and other non-MRI personnel are screened before entering the following two zones. Zone 3 has total supervision of MRI personnel and it is the transition for the entrance to the equipment room - zone 4. Patients with implantable medical devices must be well screened before undergo to magnetic resonance examination, due to potential effects and hazards from the different electromagnetic fields used in magnetic resonance imaging. So, better knowledge on safety zones and different standards are vital for safety practices both for patients and workers.
\end{abstract}

Keywords: Magnetic resonance; Occupational; Safety zones; Standards

\section{Introduction}

Nowadays, magnetic resonance imaging (MRI) is widely accepted in Medicine. However, the use of this imaging technique opens new questions regarding the hazards and new safety standards are discussed. There are potential risks in MRI environments, not only for patients and workers, but also for patients' family members, other healthcare professionals and others who find themselves only occasionally or rarely in MRI environments, like housekeeping personnel, policemen. The increase of MRI exams and the advances of this technique demand the definition of safety zones and standards. In the last decade, many advances on MRI brought new and more sophisticated equipments, allowing better accuracy and quality on diagnosis $[1,2]$. With these improvements, static magnetic fields (SMF) strengths used in MRI become higher, increasing effects and worries associated.

For MRI functioning, MRI requires three types of electromagnetic fields (EMF) [2-6]:

a. A SMF with a given field strength (from 0.2 Tesla (T) to 7T in clinical use);

b. Time-varying magnetic field gradients (TVGF), with a variable intensity and slew rate;

c. Radiofrequencies (RF) fields, which works as a transmitter and receiver system. 
All EMF used in MRI have different types of risks, hazards and side effects associated (Table 1) $[2,3,7,8]$. All these EMF used in MRI have exposure limits. For these reasons, standards and

Table 1: Effects and hazards from EMF used in MRI.

\begin{tabular}{|c|c|}
\hline Electromagnetic Field & Effect and Hazard \\
\hline Static Magnetic Field & $\begin{array}{c}\text { Attraction forces ("missile effect"); deflection or malfunction of medical devices; magneto-hydrodinamic effect; head- } \\
\text { aches; dizziness; visual disturbances; vertigo; magnetophosphenes; nausea; metallic taste; }\end{array}$ \\
\hline Time-varying Gradient Fields & $\begin{array}{c}\text { Headaches; vertigo; peripheral nerve and muscle stimulation; burns; dizziness; magnetophosphenes; nausea; MRI } \\
\text { acoustic noise }\end{array}$ \\
\hline Radiofrequencies Fields & RF deposition; tissue heating \\
\hline
\end{tabular}

Table 2: Four conceptual MRI safety zones and associated hazards.

\begin{tabular}{|c|c|c|}
\hline ACR Zones & Occupants & Hazards \\
\hline Zone 1 & Public in general & Negligible MRI hazards \\
\hline Zone 2 & Unscreened MRI patients & Immediately outside area(s) of hazard \\
\hline Zone 3 & Screened MRI patients / personnel & $\begin{array}{c}\text { Potential bio stimulation interference, access to magnet } \\
\text { room }\end{array}$ \\
\hline Zone 4 & $\begin{array}{c}\text { Screened MRI patients under constant direct supervision of trained MRI } \\
\text { personnel }\end{array}$ & $\begin{array}{c}\text { Bio stimulation interference, radiofrequency (RF) heating, } \\
\text { missile effect, cryogens }\end{array}$ \\
\hline
\end{tabular}

A MRI department has different rooms were both patients, nonMRI staff and MRI staff circulate and it is demand that those zones are well visible and detailed.

With the increasing advent and use of very high field magnet strengths in MRI, staff need to recognize that one should never assume MRI compatibility or safety information about different medical devices, if it is not clearly documented in writing.

Decisions based on published MRI safety compatibility data about an implantable medical device must respect manufacturer's specifications and MRI personnel should evaluate the risk-benefit of making a MRI exam. All patients must answer and sign a consent form and questionnaire before entering MRI examination room. Unconscious patients should be well studied before do so. Pregnant women should be well evaluated before performing a MRI examination. MRI centers need to establish, implement and maintain safety policies and procedures, routinely reviewed and updated. The proliferation of high field and ultra high MRI examination centers calls for a review of the MRI safety and standards literature.

\section{Materials and Methods}

Some search databases were used to detect important and relevant studies on MRI safety standards and their safety zones and systematic reviews on MRI effects and hazards published. A wide range of combined keywords were used, such as: magnetic resonance imaging, radiofrequency fields, static magnetic fields, time-varying gradient fields, occupational safety zones, standards, health effects, hazards, risks and safety. Only published studies with relevant conclusions and a good background description were eligible. Brief communications and not published data were excluded. occupational safety zones introduction are essential for better and safety practices because there are potential risks in a MRI environment (Table 2).

\section{Results and Discussion}

There are potential hazards and risks associated to MRI clinical environments, not only for patients and MRI workers, but also to accompanying family members, attending health care professionals, security and housekeeping personnel, etc. All clinical and research MRI sites must maintain MRI safety policies and procedures, which must be established and maintained and routinely reviewed and updated. All of procedures should be in place to ensure that any adverse events and incidents are reported [7].

As stated above, MRI uses three types of magnetic fields to create images: a) a strong SMF; b) TVGF's and c) RF fields. These types of fields bring different kinds of hazards and effects. There are been reports in medical literature detailing MRI incidents involving patients, equipment and personnel.

\section{MRI Safety Zones}

Due to the powerful SMF and TVGF used in MRI equipments, MRI departments establish four conceptual zones around MRI scanner. These safety zones were introduced by the American College of Radiology (ACR) [7]. Since the static magnetic field extends in three dimensions, some zones may extend into other areas or floors of hospital facilities. The four safety zones have personnel restrictions [7]:

a. Zone 1 (Figure 1): includes areas that are freely accessible to public and typically outside MRI environment. It is known as the access area to MRI environment. This zone is not controlled by MRI personnel;

b. Zone 2 (Figure 2): it is the interface between publicly uncontrolled accessible zone 1 and the strictly controlled zones 3 and 4. Patients and other personnel are screened in this zone, 
where MRI screening questions, patients' histories are typically obtained;

c. Zone 3 (Figure 3): all access to this zone should be strictly restricted, with access to regions within it (zone 4) controlled by, and entirely under the supervision of MRI personnel (typically the MRI technologists). This is the region in which free access of unscreened non-MRI personnel or some ferromagnetic objects and equipments can result in serious damages and injuries as the result of interactions between those equipments and SMF and TVGFs from MRI machines;

d. Zone 4 (Figure 4): this area is where MRI equipment is installed and located. Zone 4 should be clearly marked with warning panels. Avery important issue that must be considering is that the MRI SMF is always on. So, the occurrence of potential effects from SMF is always present.

In case of a cardiac or respiratory arrest or other medical emergency in zone 4, where emergent medical intervention is required, MRI staff and other health care professionals must be aware that many emergency materials are not compatible into MRI equipment room (zone 4). So, it is important that appropriately trained and certified personnel should initiate basic life support as required for the situation, while the patient is removed from zone 4 to another area, normally a magnetically safe location, such as zone 2, for example (Figures 1-4)

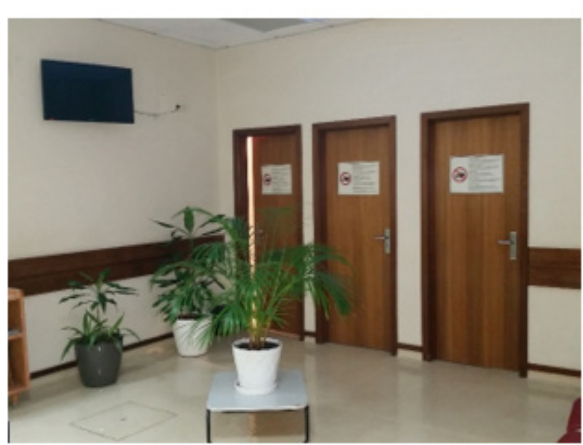

Figure1: Example of a MRI zone 1.

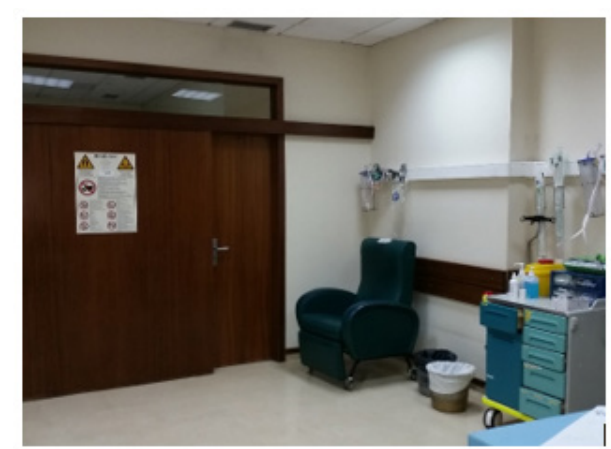

Figure2: Example of a MRI zone 2.

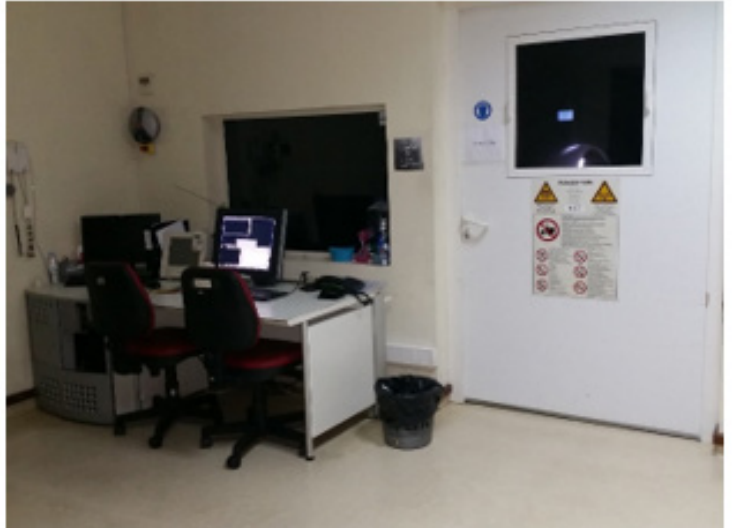

Figure3: Example of a MRI zone 3

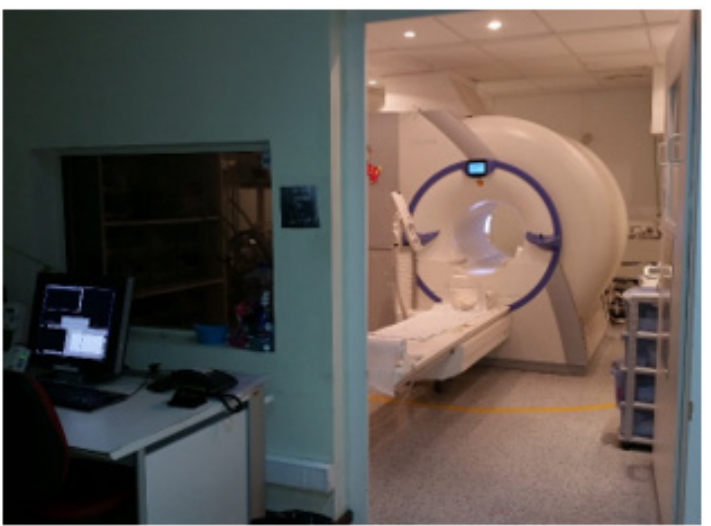

Figure4: Example of a transition between MRI zones 3 and 4

All of these conceptual MRI safety zones have different kinds of hazards, as described on Table 2 [7]. They can range between negligible hazards to bio stimulation interference with medical devices, cryogens, RF heating and attraction effects ("missile effect").

There are many implantable medical devices with may have metallic or metal components. They can be classified into two categories: i) active implantable medical devices, which include pacemakers, defibrillators, neuro stimulators, drug pumps, and others, where their functioning depends on an energy source such as electrical, mechanical or pneumatic power; ii) non-active implantable medical devices, which are passive and not require a power source for its function. In this category, joint prosthesis, heart valves, aneurysm clips, stents are examples [9].

Both of those implantable medical devices can contain metallic components that cannot be compatible with MRI equipments and therefore are contraindicated by implant manufacturer. Metallic implants can be attracted and displaced within the human body, with potentially fatal consequences. Patients with implantable medical devices must be well screened before entering MRI 
controlled safety zones.

Implantable medical devices can be categorized in other classification [9]:

- $\quad$ MR Safe: it is an item which poses no known hazards in all MRI environments;

- MR Unsafe: it is an item which is known to have hazards in all MRI environments

- $\quad$ MR Conditional: it is an item which has been demonstrated to pose no known hazards in a specific MRI environment with specified conditions of use. So, to screen a patient in MRI with an implant with this category, some equipment conditions may be required, such as field strength, specific TVGF, RF fields (Table 2).

When scanning patients with conditional implantable medical devices, radiographers must follow what manufacturer's conditions that device should perform the MRI examination safely, both for patients and workers. Nowadays, most of implantable medical devices, such as orthopaedic prosthesis, are made from nonferromagnetic material and it is safe to undergo a patient to a MRI examination.

Tattoos can be an obstacle for MRI exam. There have been some documented cases of unusual sensation or tingling from a tattoo site during MRI procedure to receiving burns or raised skin at the site. The likely hood of this happening is very low but all subjects should be aware of the issue before agreeing to make MRI exam. Nowadays, most of the inks used on tattoos are compatible, but if the subject complains of any unusual sensation during the exam the study will be immediately stopped.

\section{MRI Safety Standards}

Formation, training and knowledge on MRI safety standards are vital for better practices. By definition, there are non-MRI personnel and MRI personnel [7]. Patients and their family members, for example, are non-MRI personnel and they must pass to a MRI safety screening process to enter in zone 3 . This screening is made by MRI personnel. MRI personnel are divided into two levels: I) Level $1 \mathrm{MR}$ personnel are those who must understand MRI safety, such as the magnet is always on, basic understanding of access restriction. These include housekeeping, fire fighters, nonMRI physicians and technologists; II) Level 2 MR personnel need to have comprehensive understanding of MRI safety standards and magnetic field interaction with implants and foreign bodies. They are ultimately responsible for the screening process and have authority to independently enter in zone 4 and safely permit other to do so. They include MRI radiographers, MRI radiologists and nurses [7].

With the increasing advances and use of higher static magnetic fields, users need to recognize that one should never assume MRI compatibility or safety information about a device if it is not clearly documented in writing. Nowadays, many medical implants and devices are MRI compatible, but all patients and non-MRI personnel with a history of surgeries must undergo further investigation prior to being permitted entrance in zones 3 and 4 .

Conscious, non-emergent patients and volunteer subjects should complete and sign a written MRI safety questionnaire prior to their entrance into zone 3 and 4 . Family members that need to follow another patient (for example children) must answer the same questions as the screened patient. So, the patient, guardian or volunteer as well as the screening MRI staff member must both sign the completed safety form. These non-emergent patients must be screened on site by a minimum of two separate individuals from MRI personnel.

All patients undergoing a MRI procedure must remove all removable metallic objects and devices, such as watches, cell phones, jewellery, body piercings, cosmetics containing metallic particles (e.g. eyeliner), clothing items with metallic devices, etc. A patient with a history of a ferromagnetic foreign object penetration must be investigate too. X-ray films and computed tomography (CT) are acceptable methods of screening. For example, all patients with a history of orbit trauma by a ferromagnetic foreign body and someone who works on welding with a suspected foreign body in the orbit must be well screened before entering MRI room.

Nonresponsive and unconscious patients need to be well screened. Family members can provide medical history of those patients, such as what king of surgery was performed and when. Otherwise, the prescribing physician's exam must take responsibility of patients' screening under supervision by level 2 MRI personnel. For example, all areas of scars and deformities that might be anatomically indicative of medical implants and devices should be subject of further investigation before zone 3 and 4 entrance [7]. All unconscious patients (sedated, anesthetized or not) may be monitored during MRI examination, because thermal injuries from tissue heating from RF deposition can occur and they may not be able to express that symptoms [7].

The final determination of whether scan a patient with any implantable medical device, foreign body, tattoos is to be made by a level 2 MR personnel, such as MRI radiologist, the MRI medical director or someone designated by the latter, such as the MRI safety officer. MRI departments have their own compatible materials, such as wheelchairs, stretchers and ventilators. Non-compatible medical materials should not enter in zone 4 because of potential risks that can happen, such as attraction forces.

Some zones may extend to other areas and floors. Zone 4 is an example of that because is where MRI equipment is located. MRI scanner has a SMF with a certain field strength that produces and propagates a 5 - Gauss line which is the dispersion magnetic field 
[10]. This line is very important to consider because all implanted cardiac pacemakers, auto defibrillators, diaphragmatic pacemakers or other electrical and mechanical devices can interact with SMF, causing malfunction and damages to medical devices and consequently to patients and non-MRI personnel who have that kind of implantable medical devices. Information panels must be located where there SMF may interfere with ferromagnetic devices, indicating the field strength and what kind of objects and medical devices are in risk of incompatibility with MRI equipment. SMF is never off, so it is very important the knowledge of SMF potential effects and hazards.

Housekeeping personnel should be screened before working at MRI departments. Personnel with implanted medical devices should avoid MRI environments. These personnel need to have basic understanding on MRI safety procedures, such as the compatibility of cleaning supplies. These staffs do not possess the required training to safely enter zone 4 unattended. MRI personnel must take supervision of housekeeping personnel.

MRI scanning of prisoners with tracking electronic bracelets and handcuffs could lead to some adverse events, such as ferromagnetic attractive effects, resulting on direct injury for patients and RF interference with RF power deposition and heating of the bracelet. Therefore, in cases of scanning prisoners, they must be accompanied by authorities who will remove the devices, in spite to perform MRI exam securely.

Another safety issue is pregnancy. When heath care practitioners are pregnant they are permitted to work around MRI environment in all stages of pregnancy in zones 1, 2 and 3. The entrance in zone 4 is only permitted in emergency situations.

Pregnant patients can perform a MRI examination at any stage of pregnancy, but it is better to avoid the first trimester of pregnancy. Determination if the pregnant patient performs the MRI exam is made by level 2 MRI personnel, normally the attending radiologist. It is evaluated the risk-benefit ratio for the study. It is recommended that pregnant patients undergoing a MRI examination provide and sign a written inform consent to be sure that they understand the risks and benefits of the MRI procedure to be performed. MRI is recognized as a beneficial diagnostic tool and it is used to assess a wide range of diseases and conditions that affect pregnant, as well the foetus [11]. One of those reasons is that MRI does not use ionizing radiation, like CT scans and x-rays.

One of the side effects from TVGFs is peripheral nerve and muscle stimulation. Therefore, patients with compatible implanted and retained wires in anatomically or functionally sensitive areas (e.g. electrodes in the brain) should be consider at higher risk, especially when it is used MRI image sequences. Electrical conductive material must be removed before MRI examination. This fact is very important in intensive care unit's patients who have electrical arterial devices and non-compatible electrodes. All these kinds of materials must be removed because they function as electrically conductive materials.

Another issue brought from TVGFs is acoustic noise. For this safety aspect, hearing protection of patients and volunteers should be offered prior to undergoing any MRI examination.

$\mathrm{RF}$ deposition due to RF fields cause tissue heating and may result into burns. For example, a patient with skin ointments must clean and dry that region before MRI examination to avoid burns.

\section{Conclusion}

A clinical or research MRI center must establish, implement and maintain MRI safety policies and procedures:

- MRI sites should maintain MRI safety policies and procedures, which must be established, implemented and maintained and routinely reviewed and updated;

- If any significant change into safety parameters of MRI environment, a review and update on safety procedures should be made;

- $\quad$ Center's administration must ensure that safety policies and procedures are implemented - MRI safe practice guidelines;

- $\quad$ Procedures should be in place to ensure that all incidents and accidents are reported.

A good knowledge of safety zones, standards and practices in MRI environments can avoid many adverse events, incidents and accidents. There are risks and hazards not only for patients and MRI personnel, but also for non-MRI personnel. All patients and non-authorized personnel that require entering MRI zones must be screened before do so. All types of technology are not absolutely safe, bringing new paradigms with regard to safety aspects and a better knowledge is synonymous of good practices.

\section{Acknowledgement}

None.

\section{Conflicts of Interest}

No conflicts of interest.

\section{References}

1. Cavon ID, Glover PM, Bowtell RW, Gowland PA (2007) Tresholds for Per-ceiving Metallic Taste at High Magnetic Field. J Magn Reson Imaging 26(5): 1357-1361.

2. Crook N, Robinson L (2009) A review of the safety implications of magnetic resonance imaging at field strengths of 3 Tesla and above. Radiography 15(4): 351-356.

3. Coskun 0 (2010) Magnetic resonance imaging and safety aspects. Toxicology and Industrial Health 27(4): 307-313.

4. De Wilde JP, Grainger D, Price DL, Renaud C (2007) Magnetic resonance imaging safety issues including an analysis of record-ed incidents within the UK. Progress in Nuclear Magnetic Resonance Spectroscopy, 51: 3748. 
5. Hartwig V, Giovannetti G, Vanello N, Lombardi M, Landini L, et al. (2009) Biological Effects and Safety in Magnetic Resonance Imaging: A Review. International Journal of Environmental Research and Public Health 6(6): 1778-1798.

6. Marshall J, Martin T, Downie J, Malisza K (2007) A Comprehensive Analysis of MRI Research Risks: In Support of Full Disclosure. Can. J Neurol Sci 34(1): 11-17.

7. Kanal E, Barkovich AJ, Bell C, James P Borgstede, William G Bradley Jr, et al. (2013) ACR Guidance Document on MR Safe Practices: 2013. J. Magn. Reson. Imaging 37(3): 501-530.
8. McRobbie DW (2012) Occupational Exposure in MRI. Br J Radiol 85(1012): 293-312.

9. Samuels N (2012) Policy for the Safety of Implantable Medical Devices in Magnetic Resonance Imaging.

10. Karpowicz J and Krzysztof Gryz (2006) Health Risk Assessment of Occupational Exposure to a Magnetic Field from Magnetic Resonance Imaging Devices. Int J Occup Saf Ergon 12(2): 155-167.

11. Hand J, Y Li, E L Thomas, M A Rutherford, J V Hajnal (2006) Prediction of SAR in mother and fetus associated with MRI examination during pregnancy. Magn Resson Med 55(4): 883-893. 